\title{
Modelling and optimization of a pneumatic microfeeder system
}

\author{
Xiangdong Xue ${ }^{1, \mathrm{a}}$, Chris Bailey ${ }^{1}$, Michele Turitto ${ }^{2}$ and Svetan Ratchev ${ }^{2}$ \\ ${ }^{1}$ School of Computing and Mathematical Sciences, University of Greenwich, London SE10 9LS, UK \\ ${ }^{2}$ School of Mechanical, Materials, Manufacturing Engineering and Management, University of Nottingham, Nottingham, NG7 2RD, UK
}

Received 24 December 2008, Accepted 18 February 2009

\begin{abstract}
This paper presents modelling and design optimization of a microfeeder which, as part of a microassembly system, is used for contactless object delivery. The microfeeder consists of an array of microactuators which are controlled by electrostatic actuation and used for maneuvering outcoming air jet for object hovering and delibery. The airflow behaviour in the microactuator is analysed by means of fluid mechanics and Computational Fluid Dynamics (CFD) simulation from three aspects, theoretical analysis, initial design assessment, and design modifications. The focus is put on the basic types of the microfeeder structure and the effects of structural details to the systematic performance. The structural pattern of the microactuator for forming airflow nozzle is identified and two design plans are proposed as basic structure patterns of pneumatic microactuators. The optimized design numerically shows the ability of delivering objects. This paper analyses the flow distribution pattern in microactuators and points out a way for effective design of pneumatic microfeeder systems. The optimization strategy provided by the present paper has close relevance to the design and manufacture of pneumatic microfeeder systems.
\end{abstract}

Key words: Pneumatic microactuator, Microfeeder, Distributed manipulation, MEMS device, Actuator design

\section{Introduction}

The development of microsystems entails several integrations, respectively in space, function and physics. In space, microsystems have been developed from two dimensional to three dimensional systems. 3D is the basic form and rational pattern in the natural world. Construction of $3 \mathrm{D}$ features can thus enrich the suitability of systems and provide microsystems with extensive developing space and large design flexibility. In function, more and more active functions have been developed and performed by microsystems. These microsystems are becoming all-purpose systems moving away from passive functional based systems. In physics, mutiple domain is a distinctive character of microsystems versus macro systems. Multiphysics is widely suitable for microsystems as in the microspace, electronic and thermal are two indispensible domains. Moreover their effects have to be cosidered with other domains, such as mechanics, fluidics, magnetics, phonics and optics, etc.

In such a variety of product types, a pneumatic microfeeder using airflow as driving resource is an advanced system: it is built based on a 3D microfluidic system, concerned with multiple physics, used through active function for delivering objects by means of speed-up airflows. Arrayed microactuators are included in the pneumatic actuation system. Complex interactions, including fluidic dynamics, mechanics, electrostatics and electromagnetics, are involved in these pneumatic microactuators. Hence this pneumatic microfluidic system operates in a multiphysics environment and performs in a systematic way.

\footnotetext{
${ }^{\text {a }}$ Corresponding author: X.Xue@gre.ac.uk

There are two types of approaches with regard to contact status between the deliveried object and actuator surface [1]. One is contact delivery system that delivers an object by sliding it on the surface of the system [2]. The other is contactless system, moving an object by the interaction of a medium which acts between the device and the part that is therefore driven above the surface of the system [3]. This paper is concerned with the latter system. Comparing with a contact delivery system, the contactless system has some characteristic advantages as follows [4]: (i) Surface forces can be completely neglected; (ii) It is suitable for handling fragile, freshly painted, sensitive micron-sized structured surfaces; (iii) It allows the handling of non-rigid microparts; (iv) There is no contamination of and from the end effector. However there are more constraints for the object in order for it to be moved with no contact: the object should be planar shaped and with a balanced weight distribution.

Microfluidic systems play a key function in contactless pneumatic microfeeder systems. Microfluidic systems have specific characters from macrofluidic systems. Different from pipe systems used in macro fluidic systems, microfluidic systems are constructed with channels in solid blocks for 3D systems or in planes for $2 \mathrm{D}$ and $2.5 \mathrm{D}$ systems. Microfabrication by drilling or lithography is a method with low materials cost efficiency. However, with uniform materials and integrated structure for both channel and basement, high structural integrity and stability are obtained. Comparing with macrosystems, microfluidic flow has some peculiar characteristics. As a result of their micro size, channel walls take a large share of the cross-section of a channel, resulting in a geometry of channels with high surface-to-volume ratio. This leads to relative low flow velocity and a laminar distribution as the general flow condition [5]. With channels integrated in vulume, the wall 
surface quality in microfluidic systems is not as high as that in macrosystems where the pipe is the basic form and pipe materials and quality are more optional. Moreover, in microfluidic systems there is generally no free surface and shear stress becomes a dominant factor. It can be seen that, from a manufacturing point of view, comparably, microfluidic systems adopt a primeval natural cave form in nature, whereas macrofluidic systems generally take the advanced natural fluid system forms can that be found in living creatures.

The charateristics of microflow leads Computational Fluid Dynamics (CFD) to become an inevitable means for the performance assessment of microfluidic systems. Due to the microsize and multiple physics involved, a microfluidic system is difficult to be treated with instinct and experience. CFD therefore provides a tool for analysis of the system and an instrument for investigation and illustration of detailed fluidic performances. Similarly to other disciplinary support systems, CFD has changed from traditional assessment means and become active over all processes of products in design disposal, fabrication manipulation and reliability prediction.

This paper, presents a study on design and modelling of a micropneumatic actuator system: the part to be conveyed is suspended and moved over the surface of the device by the outcoming airflow. Relevant theoretical background of fluid dynamics is provided and structural analysis and modification are performed.. The focus of this study is put on the effects of structural features of the microfeeder to the airflow control. For this purpose, the scope is concentrated on the airflow performance with a qualitative analysis rather than a detailed integration of all the effects such as electrostatic action and mechanical deformation. The main contents of the paper are discussed through three parts, respectively on theoretical analysis, modelling \& simulation and conceptual design.

\section{Mechanism of the pneumatic actuator}

An arrayed pneumatic feeder for microassembly systems is presented in [6,7]. The array is composed of a set of microactuators, distributed in the horizontal plane. The nozzles are opened and closed by electrostatic actuation. In this way, the direction of the outcoming airflows can be controlled that is used for controlling the movement of the hovering object. Figure 1 shows the arrayed pneumatic microactuator system. The tiny actuators in the array are shaped as 3D cubic structures, each comprising a central electrode block and four side electrode walls. Four airflow nozzles, formed from the gaps between the side electrodes and central electrode, are located in the four sides of the actuator. In addition to the electrodes, there are four pillars located at the four corners of the actuator. The pillars are designed as fixed objects on the array for giving the whole structure a higher mechnical robustness. Figure 2 gives a description of a single microactuator: pressurised air is supplied from the bottom of the actuator and escapes through the nozzles located at the top of the device. The fast escaped directional air jets produce high pressure at the bottom of the object, driving it above the array and moving towards one direction parallel to array surface.

The elements of a microactuator are connected to a PCB board so that an electrostatic force can be created (Fig. 2).
Hence, the microactuator has four working and one neutral mode. Figure 3 shows the top view of a microactuator in neutral and working modes, respectively. In the neutral mode, the four nozzles are opened in equal width and the four outcoming airflows produce an equilibrium resultant force that supports the object making it hovering above the array.

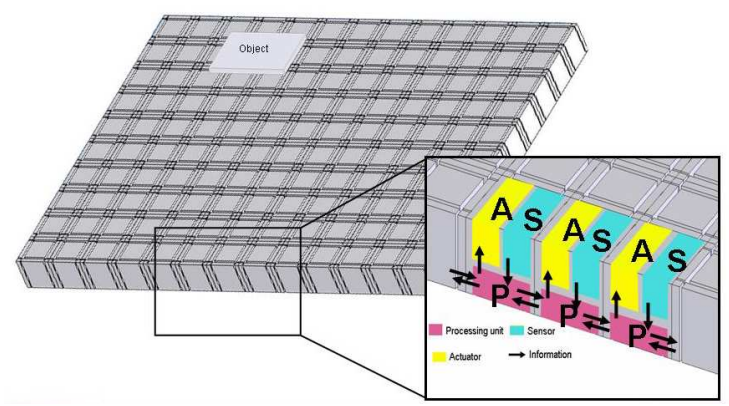

Fig. 1. Arrayed pneumatic microactuator system [6]
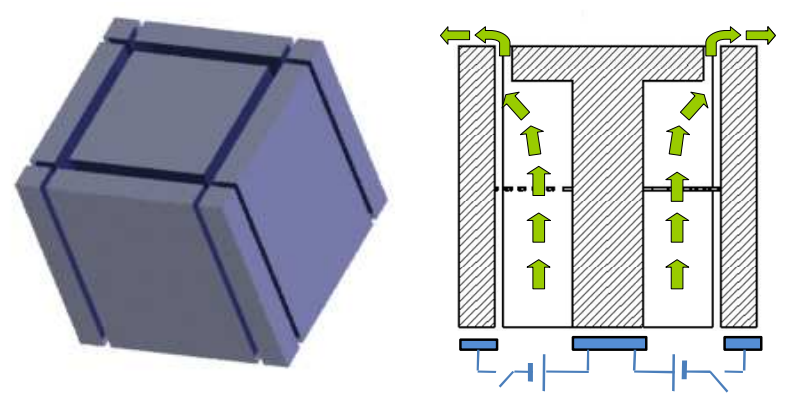

Fig. 2. Pneumatic microactuator [6]

When in working mode, electric voltage is applied to the central element and one selected side wall; the two act as the armors of a capacitor and move towards each other due to the electrostatic interaction. As a result, one nozzle is closed and another is enlarged. This leads to the equilibrium of the airflow forces in this direction being broken. With oblique collision of air jet to the object, an actuating force parallel to the feeder surface is produced correspondingly. By simultaneously controlling the actuators located under the object in a same phase, a resultant force is formed to drive the object. As microactuators are arranged in the array in two perpendicular directions, the pneumatic microactuator system can produce driving forces to move an object in two perpendicular directions.
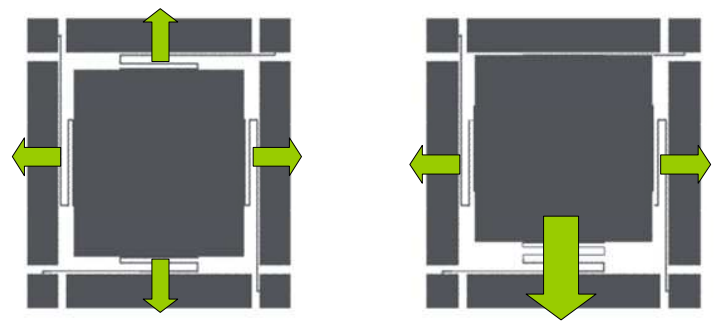

Fig. 3. Top views of a microactuator in neutral and working positions

The central electrode is not in contact with the side electrodes or the corner pillars in neutral mode. To keep a stable state of the central electrode, suspensions/springs are 
implemented between the central electrode and four side pillars. Unlike macrosystems where suspensions/springs are shaped as spiral state in $3 \mathrm{D}$, microsprings are generally constructed by some simplified methods and structures with 2D method [8], due to the difficulties in microfabrication techniques and limited space in the system. In this design, simplified springs with bending beams are adopted to form the suspensions.

The direction of the interaction force between air jets and an object depends on the direction of the air issuing from the microfeeder, whereas the air jet directions are mainly determined by the structure of the outlet nozzles. In this study, several plans are tested corresponding to different relative vertical positions between central and side electrodes to optimise the performance of a directional nozzle. Figure 4 shows the neutral mode when the central and side electrodes are positioned at the same height. Considering the symmetric character of the microactuator in two perpendicular horizontal directions, each actuator is apt to be described with a $2 \mathrm{D}$ model. With regard to the working processing of the actuator, two positions are modelled to represent the neutral and working modes, respectively.

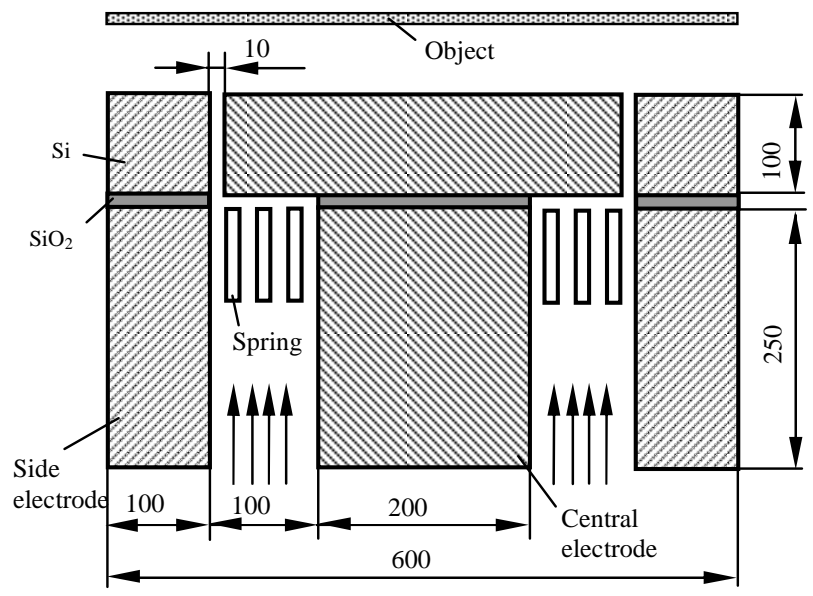

Fig. 4. Original design of the pneumatic actuator. Dimentions in the figure are in micrometers

Incompressible Newtonian flow is adopted in modelling. All contact surfaces of the airflow with solids, including the boundary surfaces of the central electrode, the inside surfaces of the side electrodes, the bottom surface of the object, as well as the suspension/spring surfaces, are modelled with walls, on which airflows are set to no-slip status. According to work conditions of the system, the input condition of airflow is determined by air pressure applied on the inlet. The outlet boundary conditions are also set to air pressures, applied at the boundary interfaces between the object and the top surface of actuator. In all circumstances, the inlet air pressure is set to $8 \mathrm{KPa}$ and the outlet air pressure is set to 0 . The detail process of the electrode motions under electrostatic force is not modelled. Instead, airflows in two extreme positions of the electrodes are analysed.

\section{Fluid dynamics of airflow}

\subsection{Conservation laws related}

The flow is considered as a steady and fully developed flow and satisfies the conservation laws. The mass conservation law of a flow can be expressed as [9]

$$
\frac{\partial \rho}{\partial t}+\operatorname{div} \rho v=0
$$

where $\boldsymbol{\rho}$ is the density of the flow and $\boldsymbol{v}$ the velocity. The conservation of mass reflects the continuity property of flow materials and denotes the relationship of the velocity and cross section of a inside flow. For imcompressible flows, $\partial \rho / \partial t=0$. Thus, Eq. (1) can be simplified as

$$
\operatorname{div} \rho v=0
$$

The conservation of momentum of a flow can be expressed as

$$
\frac{\partial(\rho v)}{\partial t}+\operatorname{div}(\rho v v)=\operatorname{div} T+\rho f^{b}
$$

where $\boldsymbol{T}$ is the stress tensor of the flow and $f^{b}$ the body force. The conservation of momentum reflects the application of Newton's second law of motion in the region of fluid dynamics, implying that the change rate of momentum of material control volume, in time and in space, equal to the forces applied on the volume, by body forcea and tension stress, respectively.

When supplied through the inlet until it strikes the planar object, the airflow experiences several stages. Firstly, it passes through the cavity. Then, it rushes through the outlet nozzle. Finally, it strikes the bottom of the object. In the following subsections, these processes are analysed using conservation laws.

\subsection{Airflow channel}

The changes of the airflow velocity between the inlet cavity inside actuator and the outlet channel can be deducted from mass conservation. Exhibited with integral form, the mass conservation Eq. (2) can be expressed as,

$$
\int_{\text {chan }} \rho V_{y_{-} \text {chan }} d A_{\text {chan }}=\int_{\text {cell }} \rho V_{y_{-} \text {cell }} d A_{\text {cell }}
$$

Or,

$$
\int_{A_{\text {chan }}} V_{y_{-} \text {chan }} d A_{\text {chan }}=\int_{A_{\text {cell }}} V_{y_{-} \text {cell }} d A_{\text {cell }}
$$

where, $\rho$ is the flow density, $V$ the flow velocity, $A$ the cross area. The subcripts chan and cell denote the outlet channel and inside cavity, respectively. Using average quantities for the velocity, Eq. (5) can be expressed as

$$
V_{y_{-} \text {chan }, \text { avg }} A_{\text {chan }}=V_{y_{-} \text {cell }, \text { avg }} A_{\text {cell }}
$$

That is,

$$
V_{y_{-} \text {chan }, \text { avg }} / V_{y_{-} \text {cell }, \text { avg }}=A_{\text {cell }} / A_{\text {chan }}
$$

It can be seen from Eq. (7) that the average velocities in the outlet channel and inside cavity are inversely proportional to the cross-area. In this design, the area of the cross-section of the inside cavity is ten-times that of the outlet channel. The velocity in the outlet channel is therefore much higher than that in the inside cavity.

\subsection{Outlet nozzle}


The pressure loss when the airflow passes through the outlet nozzle can be analysed using the momentum conservation law. Having a steady flow, $\partial(\rho v) / \partial t=0$. Substituted into Eq. (3) and expressed with integral form, Eq. (3) becomes:

$$
\begin{aligned}
& \int_{A_{\text {chan }}} \rho v_{\text {in }}^{2} d A_{\text {chan }}-\int_{A_{\text {chan }}} \rho v_{\text {out }}^{2} d A_{\text {chan }} \\
& =\left(P_{\text {in }}-P_{\text {out }}\right) A_{\text {chan }}-\tau_{w} A_{\text {wall }}-\rho g L A_{\text {chan }}
\end{aligned}
$$

where $P$ is the pressure, $\tau_{w}$ the shear stress, $A_{\text {wall }}$ the cross section for the wall of the outlet nozzle, $L$ the length of the nozzle, the subscripts in and out denote the entering and escaping of airflow from a control volume.

From conservation of mass,

$$
\int_{A_{\text {chan }}} \rho v_{\text {in }}^{2} d A_{\text {chan }}=\int_{A_{\text {chan }}} \rho v_{\text {out }}^{2} d A_{\text {chan }}
$$

Eq (8) becomes:

$$
\left(P_{\text {in }}-P_{\text {out }}\right)-\rho g L=\tau_{w} \frac{A_{\text {wall }}}{A_{\text {chan }}}
$$

For a nozzle with rectagular cross-section of width a and length $b$,

$$
\frac{A_{\text {wall }}}{A_{\text {chan }}}=\frac{2(a+b) L}{a b}
$$

For a nozzle with square cross-section of edge length a,

$$
\frac{A_{\text {wall }}}{A_{\text {chan }}}=\frac{2(2 a) L}{a^{2}}=\frac{4 L}{a}
$$

For a nozzle with circle cross-section of diameter D,

$$
\frac{A_{\text {wall }}}{A_{\text {chan }}}=\frac{\pi D L}{\frac{1}{4} \pi D^{2}}=\frac{4 L}{D}
$$

Substitute these into Eq.(9), one has

$$
P_{\text {in }}-P_{\text {out }}=\tau_{w} \frac{4 L}{D_{\text {cross }}}+\rho g L
$$

where $D_{\text {cross }}$ is the character parameter of cross-section of the nozzle. For a circle, $D_{\text {cross }}=D$, for a square, $D_{\text {cross }}=a$, for a rectagular, $D_{\text {cross }}=\frac{2 a b}{(a+b)}$.

In microflow, viscous forces are far larger than inertial force, becoming a dominant factor. Ignoring gravitional function, Eq. (10a) becomes

$$
P_{\text {in }}-P_{\text {out }}=\tau_{w} \frac{4 L}{D_{\text {cross }}}
$$

It can be seen from Eq.(10) that pressure drop through a nozzle is decided by the length and cross section of the nozzle. As the cross section of the inlet cavity is much larger than that of the outlet nozzle, pressure loss in the inlet cell is thus much less than in the nozzle. It can also be seen that the solution to pressure loss is transferred to searching wall shear stress. From fluid dynamics,

$$
\tau_{w}=\left.\mu \frac{\partial v_{x}}{\partial y}\right|_{y=a / 2}
$$

where $\boldsymbol{\mu}$ is the dynamic viscosity, $\boldsymbol{v}_{\mathrm{x}}$ the velocity along the axis direction of the nozzle, $\boldsymbol{y}$ the distance in ratius direciton of the nozzle and $a / 2$ the distance from flow centre to nozzle wall.

\subsection{Interactions of airflow with the object}

Figure 5 shows the interaction between the issuing airflow and the conveyed object. The airflow rushes out from the outlet nozzle in an oblique direction, striking on the bottom of the object. When this airflow jet strikes on the solid object, the particles of airflow do not rebound but instead flow over the bottom surface of the planar object [10]. For the directional outcoming air jet shown in Fig. 5, most of the flow will travel towards the right-hand side.

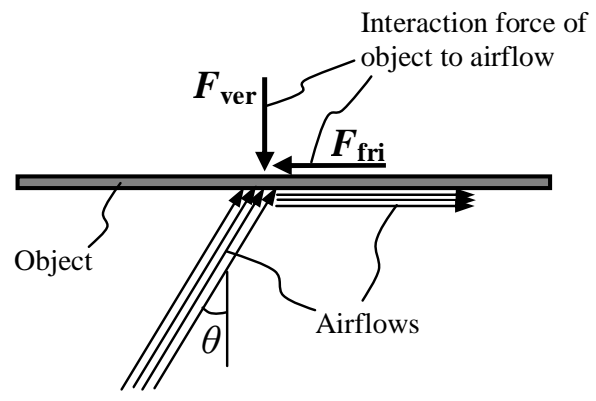

Fig. 5. Interaction between a planar object and airflow

The interactions between the airflow and the bottom of the object can be analyzed using the conservation of momentum. In the vertical direction of the planar object, the interaction follows Newton's second law of motion:

$$
\frac{d\left(m v_{v e r}\right)}{d t}=\sum F_{v e r}=F_{o b j}+F_{b o d y}
$$

or

$$
\sum F_{v e r}=m \frac{v_{v e r, 1}-v_{v e r, 0}}{\Delta t}=m \frac{v_{v e r, 1}-v_{0} \cos \theta}{\Delta t}
$$

where $\boldsymbol{m}$ is the mass of air particles, $\Delta \boldsymbol{t}$ the action period the flow striking the object, $v_{0}$ and $v_{1}$ the velocities of the airflow just before and after the interaction, $\sum F_{v e r}$ the resultant force acting on the airflow in the vertical direction, including the interaction force of the object to the airflow $F_{o b j}$ and the gravity force of the airflow $F_{b o d y}$. As the reflect vertical velocity of the airflow $v_{v e r, 1}$ becomes zero, Eq. (12) becomes

$$
F_{o b j}=-m \frac{v_{v e r, 0}}{\Delta t}-m g=-m\left(\frac{v_{0} \cos \theta}{\Delta t}+g\right)
$$

$F_{o b j}$ equals to the force acting on the object, which is used to support the object hovering above the microfeeder's surface. The acting time $\Delta t$ is related to the stiffness of the object surface.

In the horizontal direction, the momentum interaction can be expressed as

$$
\frac{d\left(m v_{h o r}\right)}{d t}=\sum F_{h o r}=F_{f r i}
$$

or 


$$
F_{f r i}=m \frac{v_{h o r, 1}-v_{h o r, 0}}{\Delta t}=m \frac{v_{h o r, 1}-v_{0} \sin \theta}{\Delta t}
$$

It can be seen that through the impact interaction of the airflow with the object, the momentum of the airflow is changed from an oblique to horizontal direction. This implies that all the momentum in the vertical direction is transmitted to the force to support the object, whereas most of the momentum in the horizontal direction is remained. The difference between the original and final momentums in horizontal direction is transmitted into the friction force driving the object.

\section{Analysis and design of outlet nozzle}

Airflow direction is closely related to the geometry of the outlet nozzle which is formed by the gap between the central and side electrodes. The original design of the outlet nozzle is assessed and optimised through the analysis of the effects of relative positions between central and side electrodes on the outcoming airflow direction.

The original design of the microactuator has been shown in Fig. 4, in which the upper edge of the central and side electrodes are located at the same height. Finite volume method is used in the modelling. Three analysis packages, Physica [11], Ansys [12] and Comsol [13], were used in the simulation of this case to verify the suitability of different packages. Consistent results are obtained among these three packages. The maximum airflow velocities of the microactuator in the outlet channel obtained by the packages Ansys, Physica and Comsol are $21.0 \mathrm{~m} / \mathrm{s}, 21.4 \mathrm{~m} / \mathrm{s}$ and 23.0 $\mathrm{m} / \mathrm{s}$, respectively. Figure 6 shows the velocity vector profile of the airflow simulated with Physica.

It can be seen that the microactuator transmits pressurised air into accelerated air flows, which are used as the driving resource to support or move the object. The high pressure air is provided from wide sized inlet to keep a stable input of air resource. Passing through the narrow shaped outlet nozzle, airflows are concentrated and speeded up. The escaped highspeed airflow rushes out of the outlet nozzle, striking the bottom of the object.

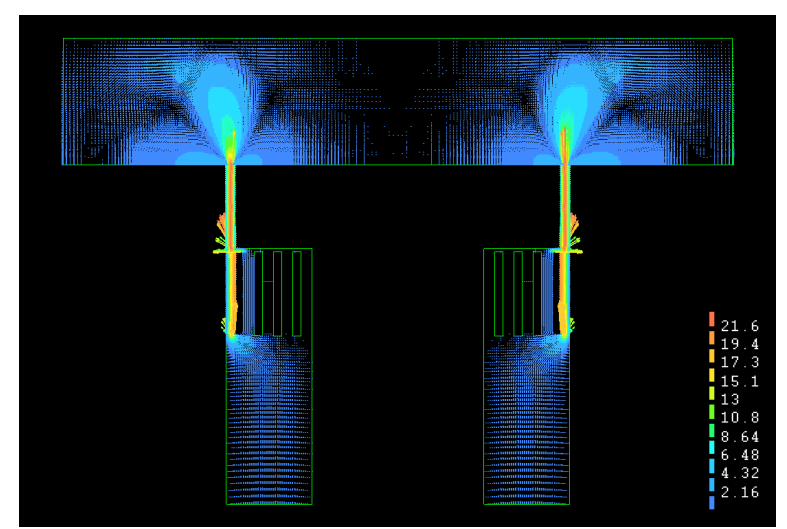

Fig. 6. Velocity vector profile of micro airflow in the neutral position

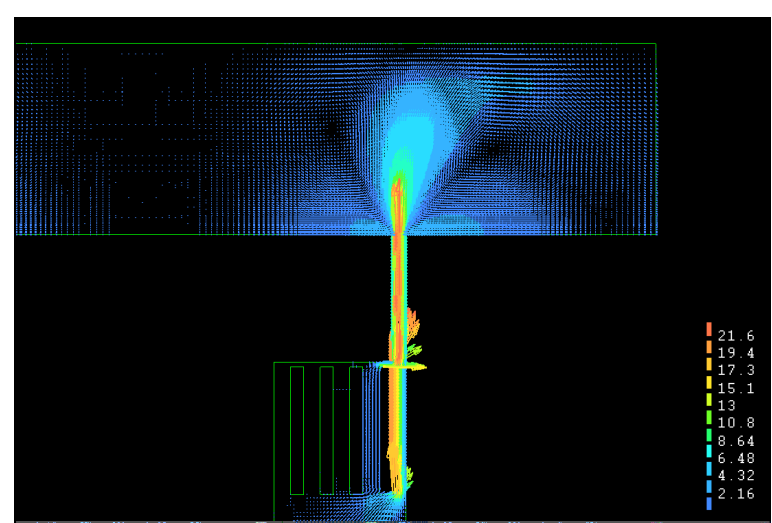

Fig. 7. Outcoming airflow in the plan when the central and side electrodes located at the same heights

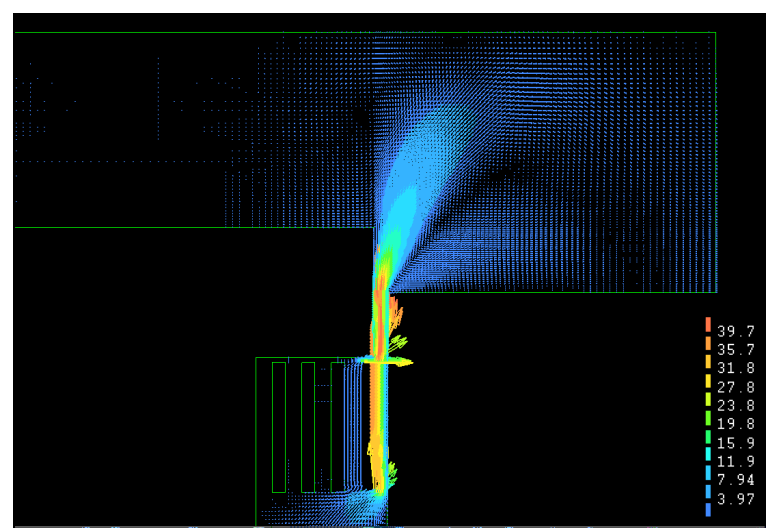

Fig. 8. Outcoming airflow in the plan when the upper edge of the side electrode being lower than that of the central electrode

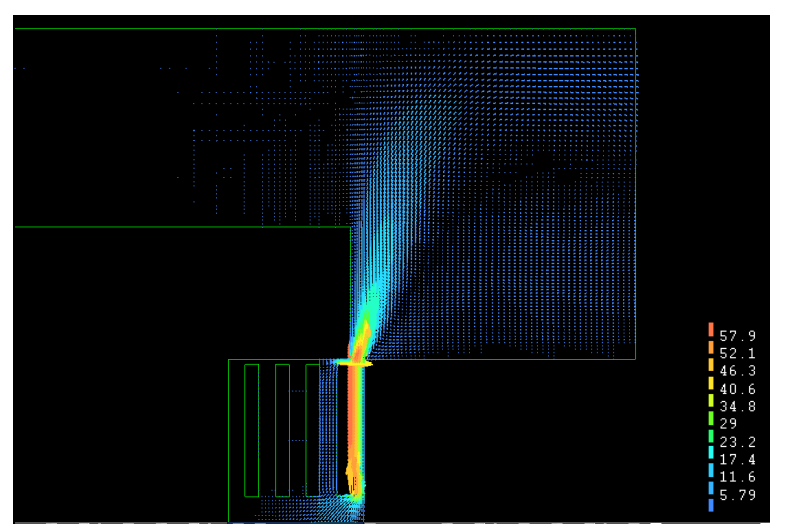

Fig. 9. Outcoming airflow in the plan when the upper edge of the side electrode located at the same height as the below edge of the central electrode 


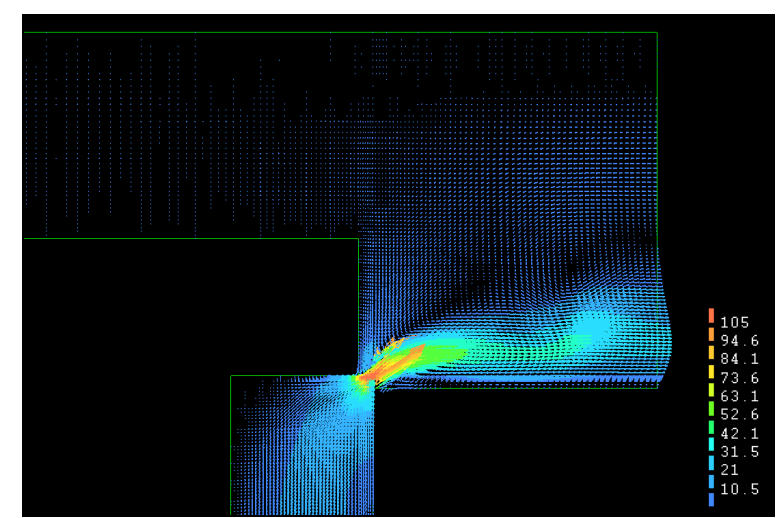

Fig. 10. Outcoming airflow in the plan when the upper edge of the side electrode is lower than the below edge of the side electrode

Four locations corresponding to different relative heights of the central and side electrodes were modelled to investigate their effect on the air jet directions. The velocity vectors are shown in Figs 7 through 10.

Figures 7 to 10 show that the outcoming direction and development of the airflow are affected by the following factors: the normal direction of outlet nozzle channel/wall located outside of the nozzle, and the geometry and air pressure in the cavity between two electrodes. It can be seen that when lowering the height of the side electrode, the deflection angle of the airflow incresed. The lowering side electrode brings two changes to the local structure. One is that a side wall is formed by the side surface of the top part of the central electrode. The other is that the normal direction of the outlet nozzle is changed. It is in the vertical direction in Figs 7 and 8 and changed to oblique directions in Figs 9 and 10.

It can also be seen that the velocity of the outcoming airflow is roughly inversely proportional to the length of the outlet channel. The maximum velocity of airflow is $22 \mathrm{~m} / \mathrm{s}$ in the case of full length channel (Fig. 7), 40m/s in the case of half-length of the original channel (Fig. 8) and $58 \mathrm{~m} / \mathrm{s}$ in the case without channel (Fig. 9). This is coped with Eq. (10), in which the pressure drop over outlet channel is roughly inversely proportional to the length of the channel.

Based on the simulation results, the initial design with the central and side electrodes at same height (Fig. 6 or 7) cannot provide an oblique air jet and is thus abandoned. The structures shown in Figs. 8 to 10 can produce oblique air jets and thus are suitable options. Among these, the oblique nozzle configuration shown in Fig. 10 has the advantage of providing flexible adjustment for the air jet direction and is therefore adopted.

The effects of various factors on the airflow distribution are discussed in detail as follows:

- Nozzle normal direction. This determines the normal direction of the outcoming airflow and is the most important factor for the basic direction of the airflow. It can be seen from Fig. 10 that by varying the normal direction of the nozzle from vertical to an oblique angle, the escaped direction of the airflow is dramatically changed.

- Walls located outside of the nozzle. This affects and deflects the moving direction of airflow escaped from the nozzle. A vertical wall located just outside of the nozzle can prevent the airflow to deflect towards one side and lead the flow to diffuse towards the other direction. It can be seen from Figs 8 and 9 that the side surface of the top edge of the central electrode and the top surface of the side electrode constitute right angled walls to regularise the airflow development.

- Channels. Outlet channels have the function of concentrating and regulating the airflow direction [14]. Passing through a channel, the distribution of the air in the channel becomes even and the flow direction is focused. However, with high resistance in a microchannel, the kinetic energy of the airflow is reduced (see Eq. (10)). It can be seen from Figs 6 and 7 that the maximum air jet velocity is doubled when the outlet channel length becomes a half of the original one.

- Air outlet route. Airflow finally goes out between the bottom of the object and the top surface of the actuator. This determines the development trend of the airflow in the final stage. As the airflow movement is determined by its kinetic momentum in the early stages, the movement of the airflow in the late stages is decided by the differences of air-pressures in the system.

- Air inlet cavity between electrodes. These should ensure the stable supply of pressurised air and satisfy other requirements, such as fabrication, mechanical and electrostatic performances. A low velocity and high pressure air is suitable as it can keep continuity supplies and so obtain a stable performance of the feeder.

\section{Conceptual design of microactuator}

\subsection{Categorizing patterns of microactuator}

Being the driving force, the airflow direction is the focus in the microactuator design. As discussed above, the airflow distribution is mainly affected by the following factors: $(i)$ nozzle normal position, (ii) outside walls of nozzle and (iii) outlet channel axial directions. The normal direction of the nozzle is formed by the two convexes of the two top edges of the central and side electrodes. By adjusting the relative position of the central and side electrodes, different angles of the nozzle can be formed. The relative position of the walls outside of the nozzle is related to the relative heights of the central and side electrodes. If located at the same height, the normal directions of two walls are towards one direction. If located at different heights, the top surface of one electrode and the side surface of the other electrode constitute two perpendicular walls outside of the nozzle. In this case, these two walls can regulate the outcoming airflow direction. Unlike the wall outside of the nozzle, the walls inside the nozzle in the cell are always positioned in perpendicular angles and thus the airflow in the cavity of the actuator is decided by the width and height.

To form an oblique directional nozzle and two perpendicular walls outside and inside the nozzle, the central and side electrodes should be located at different heights and one of the central and side electrode has to be shaped as a angle. There are therefore two possible shapes of electrodes, i.e. angle and wall, and two relative positions of the central and side electrodes, i.e. whichever electrode with the angle structrue located higher than the other. Figure 11 shows these 
two basic plans in neutral position, in which the nozzles are formed by two top convexes of the electrodes. Plan (a) is discussed in the present paper, whereas plan (b) has been processed by literature [3].

Embodying an outlet channel can effectively control the direction and concentration of an air jet. For a nozzle design with embodied a channel to increase the concentration of the airflow, the axial direction of the channel should not in the vertical direction so that a horizontal branch driving force can be produced. The design plans with embodied outlet channel is developed from Fig. 11, shown in Fig. 12. In this way, the outcoming airflows are more concentrated in a fixed direction, no matter in a neutral or working mode. With an embodied channel, the oblique surfaces on the central and side electrodes are also easy to be equipped with each other in a working mode. However, a more complex fabrication required for this option that, at present state, is a challenge technique.

It can be seen that two categories of the microactuator have some similarities: both have oblique nozzles and two perpendicular walls extending out from the nozzles. The geometries inside the nozzles are also similar. Both designs can therefore produce similar directional air jets rushing out from nozzles. The differences lie in deflected directions of airflows. The airflow directions outcoming nozzles in Plan A are towards two sides, whereas in Plan B towards the central region. As a result, the two flows in Plan B coming from the same actuator will interact with each other and two flows in plan A may interact with the flows from other adjacent units. Correspondingly the acting place of the resultant airflow force on the object in Plan B is located above the unit and in Plan A is above unit boundaries.

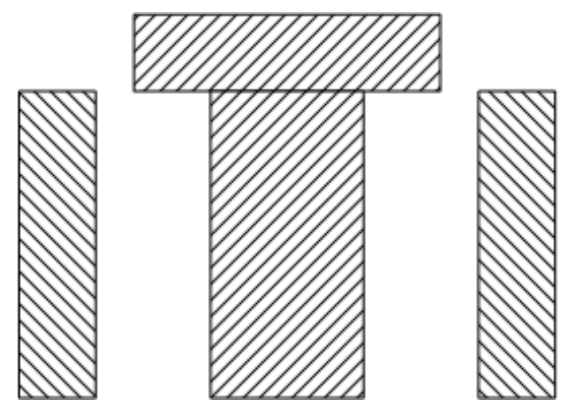

(a)
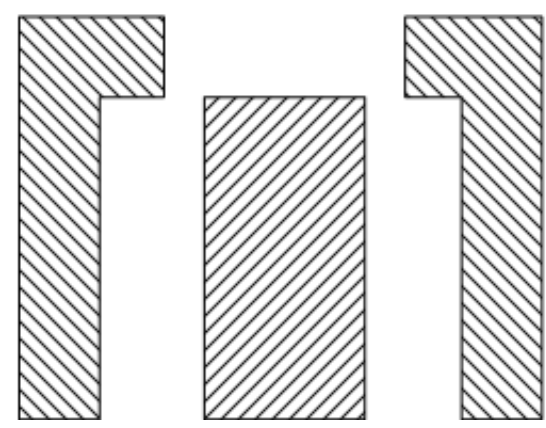

(b)

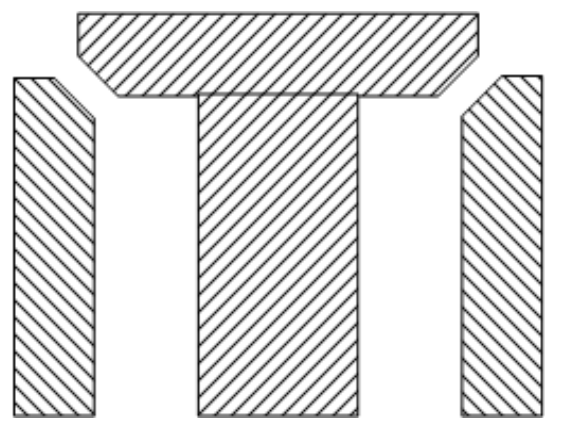

(a)

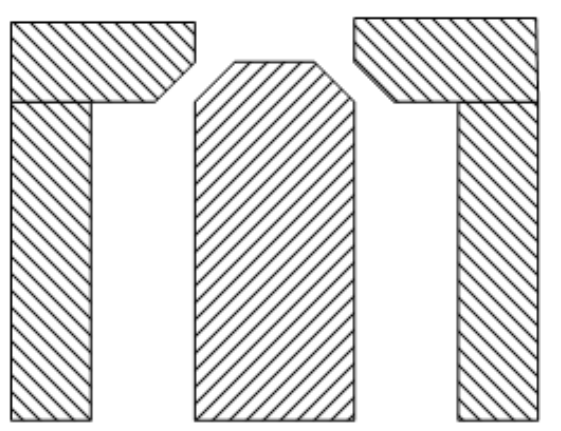

(b)

Fig. 12. Alternative designs of outlet nozzles with channels embodied

\subsection{Performance of microactuator of Plan A for this design}

Based on the above discussions, the concept design of Plan A is chosen and simulated. The velocity vector profiles are shown in Figs 13 and 14. Both figures show oblique directional outlet nozzles. The difference between the two options is that short channels are embodied in the outlet nozzle in Fig. 14. That is, Figs 13 and 14 are corresponding to the Plan A in Figs 11 and 12, respectively. The angle of elevaton of the outlet nozzle in Fig. 14 is $60^{\circ}$. It can be seen that the two designs work well with regard to airflow distribution. Between the two options, the case with an oblique channel nozzle results in more concentrated airflow. An oblique directional airflow is produced. It strikes the bottom of the object, producing both vertical and horizontal forces for hovering and driving the object.

In working mode, the airflow distribution in an actuator is not symmetric anymore as in neutral mode. Considering the airflow direction, two halves of adjacent neighbouring actuators are chosen for modelling for working mode. Figures 15 and 16 show the velocity vectors of the simulation of the working mode. It is assumed that in working mode, the airflow flows from one nozzle. It can be seen that with the size of outlet nozzle widening, the mass flow rate of the airflow is larger in work mode than in neutral mode. It can also be seen that the structure with embodied short channels, i.e. in Fig. 16, can be more effective at directing the airflow.

Fig. 11. Two basic designs of air outlet nozzle 


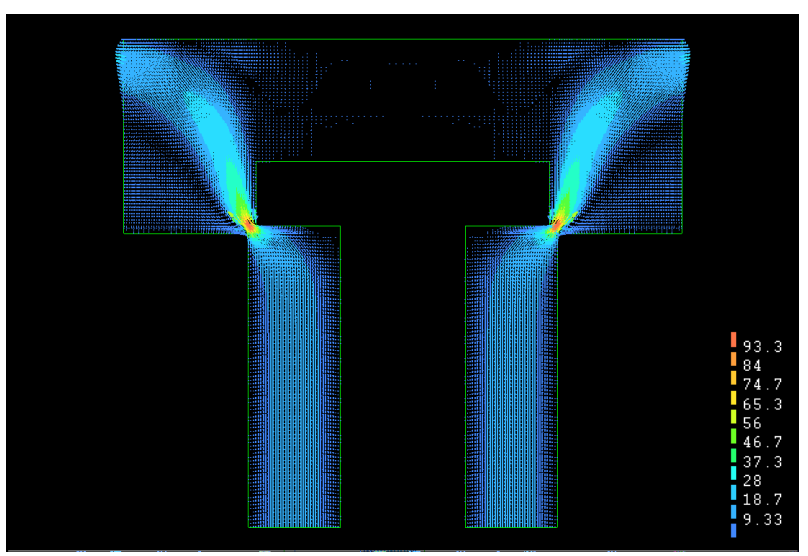

Fig. 13. Velocity profile of the modified design in the neutral mode without nozzles

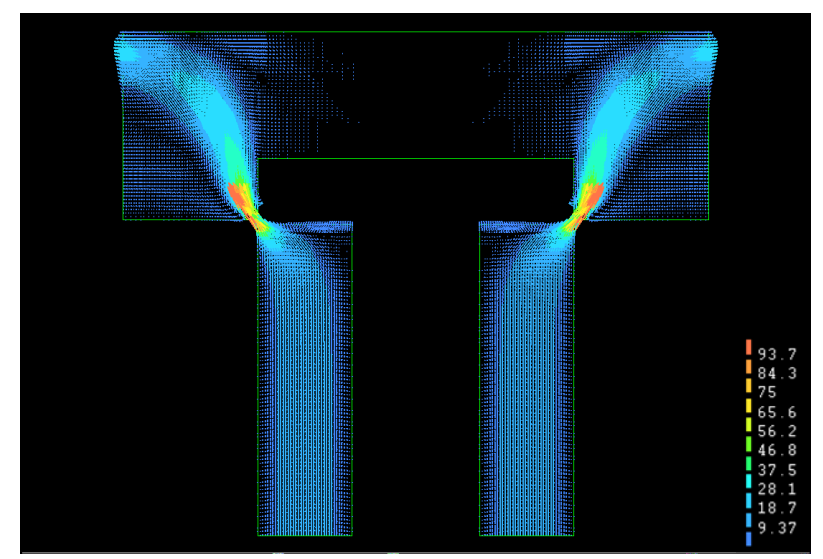

Fig. 14. Velocity profile of the modified design in the neutral mode with embodied outlet nozzles

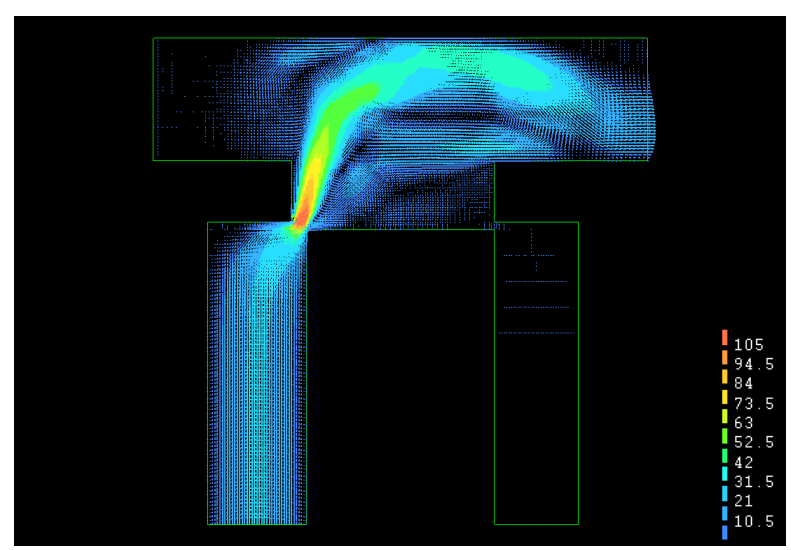

Fig. 15. Velocity profile of the modified design in a working mode without outlet nozzles

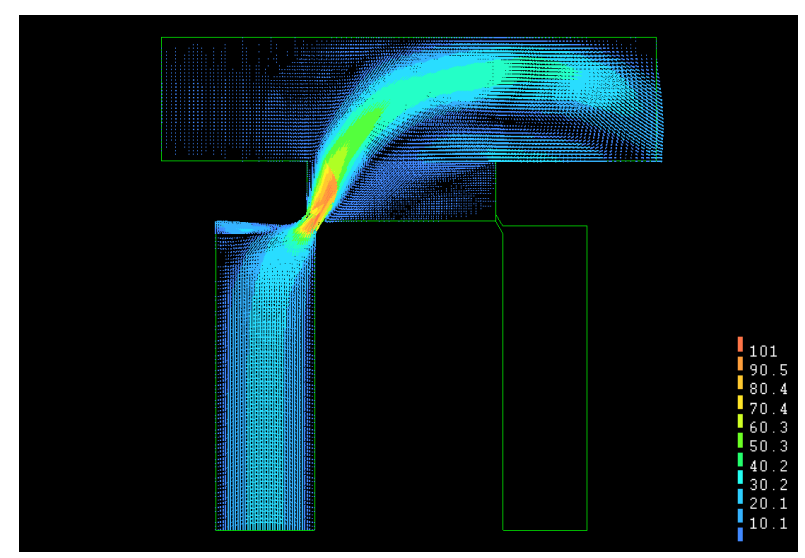

Fig. 16. Velocity profile of the modified design in a working mode with embodied outlet nozzles

After abandoning the long upright outlet channel used in the original design and adopting an oblique directional nozzle, the direction of the outcoming airflow is more controllable and the velocity of the airflow is increased. This results in a more flexible suitability of the feeders and a more effective action of the airflow.

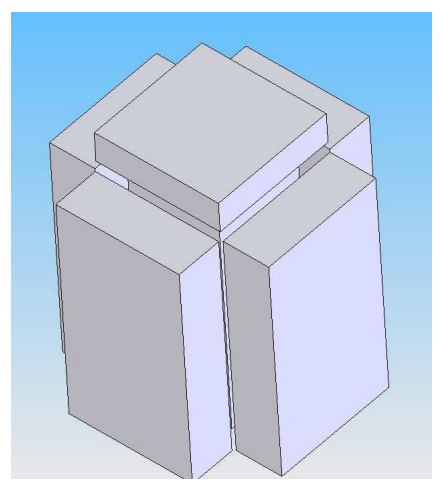

Fig. 17. A prototype of the new design

The initial microfeeder design has been modified on the basis of the results from the CFD simulations. Such changes have allowed an improvement in the device performance in directing the air towards the desired directions. Based on the result, a prototype shown in Fig. 17 is being built at the University of Norttingham. The corresponding relevance will be reported in due course.

\section{Conclusions}

Multidisciplines of electric, mechanical and fluidic are concerned with the microfeeder and fluidic dynamics behaviour is the key factor for the system performance. Electric mainly plays control roles to apply the electrostatic force on electrodes of the units and also for the coordination of the arrayed system. Mechanical functions are involved in the movement of electrodes to control the states of airflow nozzles and to undertake the task driving an object. Fluidic dynamics is used for performing actuation function. Under the actions of electric and mechanic effects, the distribution and momentum of the airflows are controlled to such a state that their interactions with the bottom of the object will produce appropriate air-pressure force to hover and drive it to the desired position. 
The development of the airflow in the microactuator can be divided into three stages: ( i) inputing into inlet cavity, (ii) speeding-up through nozzle and (iii) interacting with the delivered object. The inlet cavity should sustain a constant high pressured air as the action resource of the microactuator. The cavity should not be designed too narrow for this reason and by such the resistant force to airflow can also be reduced. The nozzle is the key link for producing directional airjet. Its performance is closely affected by geometry constitution around the nozzle. The interaction between the airflow and the object is determined by airjet direction and velocity. The vertical branch of the momentum of the airjet produces the hovering force and the horizontal branch creats the driving force for moving the object.

The geometry of a nozzle is decided by the normal direction of the nozzle, the walls extending from the nozzle and the nozzle structure, i.e. whether embodying with or not a channel. The outcoming direction of the airflow is mainly determined by the normal direction of the nozzle. The extending walls outside the nozzle have the function of enhancing the airflow direction. With two perpendicular shaped walls extending from a nozzle, the airflow can be controlled so to have a significant horizontal speed component. The effect of the air exit route is related to the final travel trend of the airflow. As the airflows in this studied microfeeder flow towards two sides of the unit, interactions between airflows from two adjacent units may occur. The structure detail inside the cavity affects the stability of the air supply and the electrostatic force level. These two have contradictive requirements in many cases.

The basic structures for pneumatic microactuator are categorised into two patterns. These are corresponding to relative positions of the central and side electrodes. Both have oblique outlet nozzles and can produce directional air jet as the driving resource to move an object. For these two structural patterns, adopting embodied directional channel for outlet nozzle can increase the effectiveness of the actuators. In this situation, the outcoming airflow can be kept in a fixed direction for both neutral and working modes and so has steady performance. Anyway in this case fabrication becomes much more challenging.

\section{Acknowledgement}

The authors gratefully acknowledge the financial support by the UK Engineering and Physical Sciences Research Council (EPSRC) in funding the Grand Challenge Project '3DMintegration' (www.3D-mintegration.com), referenced EP/C534212/1.

\section{References}

1. K.F. Bohringer, B.R. Donald, N.C. MacDonald, Upper and lower bounds for programmable vector fields with applications to MEMD and vibratory plate parts feeders, In Proceedings of International Workshop on Algorithmic Foundations of Robotics, 1996.

2. T. Ebefors, G. Stemme, Microrobotics, in the MEMS Handbook, (M. Gad-el-Hak, Editor, CRC Press, 28.128.42, 2002).

3. Y. Fukuta, Y.A. Chapuis, Y. Mita, H. Fujita, Design, fabrication, and control of MEMS-based actuator arrays for air-flow distributed micromanipulation, Journal of Microelectromechanical Systems, 15(4), 912-926 (2006).

4. P. Lambert, V. Vandaele, A. Delchambre, Non-contact handling in micro-assembly: state of the art, In Proceedings of the International Precision Assembly Seminar IPAS' 2004, Bad Hofgastein, Austria, 67-76 (2004).

5. G. Karniadakis, A. Beskok, N. Aluru, Microflow and Nanoflow: Fundamentals and Simulations, (Springer, New York, 2005).

6. M. Turitto, Y.A. Chapuis, S. Ratchev, Pneumatic Contactless Feeder for Microassembly, In Proceedings of IPAS'2006 - The Third International Precision Assembly Seminar, Bad Hofgastein, Austria, published by Springer as "Precision Assembly Technologies for Mini and Micro Products", 53-62 (2006).

7. M. Turitto, S. Ratcheva, X. Xue, M. Hughes, C. Bailey, Pneumatic contactless microfeeder design refinement through CFD simulation, In Proceedings of 4M 2007, The $3^{\text {rd }}$ International Conference on Multi-material Micro Manufacture, Borovets, Bulgaria, 65-68 (2007).

8. R.M. Lin, W.J. Wang, Structural dynamics of microsystems - current state of research and future directions, Mechanical Systems and Signal Processing, 20, 1015-1043 (2006).

9. P. Wesseling, Principles of Computational Fluid Dynamics, (Springer, Verlag Berlin Heidelberg, 2001).

10. B. Massey, Mechanics of Fluids, (Taylow \& Francis, London, 2001).

11. PHYSICA - Multi-physics Simulation in the 21th century, http://www.physica.co.uk/.

12. ANSYS Inc., ANSYS Release 11.0 Documentation. (Canonsburg PA. USA, 2008).

13. COMSOL multiphysics, http://www.comsol.com.

14. N. Naik, C. Courcimault, H. Hunter, J. Berg, J. Lee, K. Naeli, T. Wright, M. Allen, O. Brand, A. Glezer, W. King, Microfluidics for generation and characterization of liquid and gaseous micro- and nanojets, Sensors and Actuators A: Physics, 134, 119-127 (2007). 